\title{
ARE METEORS A TOOL FOR STUDYING THE ASTEROIDS? OR VICE VERSA?
}

\author{
R. E. MCCROSKY \\ Smithsonian Astrophysical Observatory
}

The bulk of the comments I made at the colloquium are to be found in two recent papers (McCrosky and Ceplecha, 1970; McCrosky et al., 1971). The following summary is offered in place of a full text.

Most interpretations of all meteor data rely on knowledge of the relationship (luminous efficiency) between mass and luminosity. Discussions of recent experiments made to determine the luminous efficiency are found in papers by Ayers et al. (1970) and Becker and Frichtenicht (1971). Faint meteor phenomena can be understood if the meteoroids are weak, low-density $\left(\rho_{m} \approx 0.25 \mathrm{~g} / \mathrm{cm}^{3}\right)$ "fluff balls" such as can be expected on the basis of Whipple's comet model (Jacchia, 1955; Jacchia et al., 1967). Alternative explanations, not requiring low densities, have been offered. Jones and Kaiser (1966) propose thermal shock of strong, high-density material as a fragmentation mechanism. Allen and Baldwin (1967) and Baldwin and Allen (1968) have reanalyzed Jacchia's data in terms of phenomena observed in the laboratory in simulated reentry experiments. Here, a high-density meteoroid froths and thereafter behaves as a low-density body. They also revise the luminosity law to account for blackbody radiation of refractory material. McCrosky and Ceplecha (1970) show that neither of these alternative explanations can apply to large bodies and, using photographic observations of bright fireballs, they defend Jacchia's original proposal for all meteors.

The mechanism for producing large, low-density meteoroids is unknown. The comet model places limits on the size of the body that can be carried away by comet outgassing. Whipple (1967) suggests that large, weak material might be of asteroidal origin ("half-baked" asteroids) and perhaps closely related to, or equivalent to, carbonaceous chondrites. However, Ceplecha (1968) shows that among the small bodies there exist at least two classes, $A$ and $C$, distinguishable from the observations. Class $\mathrm{C}$ is apparently of lower density. Class A might be similar to carbonaceous chondrites (McCrosky and Ceplecha, 1970). Members of both classes are clearly associated with comets through comet-meteor shower associations. Cook (1970) expands Ceplecha's analysis and finds evidence that class A material comes from old comets or comet interiors whereas class $\mathrm{C}$ is the material from the outer shell. 
The analysis of the in-flight photographic data of the recovered meteorite, Lost City, by McCrosky et al. (1971) points out the possibility of the substantial errors in previous density determinations if bodies of the flattened shape of Lost City are common. It is not reasonable, however, to use this argument to explain every case of fireballs that seem to have structures or densities unlike meteorites.

The most accurate and/or most comprehensive list of orbits for photographic meteors are presented in the following papers: for faint meteors, papers by Jacchia and Whipple (1961), Hawkins and Southworth (1961), and McCrosky and Posen (1961); for orbits of meteors of intermediate brightness, Whipple (1954); and for fireballs, McCrosky (1968). However, the reader should also see Kresák (1970) for discussion of possible important observational biases in these data. The half dozen cases known to be high-density material (asteroidal) or with flight characteristics thought to be appropriate for such material are given, with references, in McCrosky et al. (1971).

The problem of producing Earth-crossing orbits for asteroidal material is left to another speaker. The question of whether such material is evident among photographic meteors has most recently been extensively discussed by Kresák (1969). Convincing arguments are used to divide the meteors into pure cometary (II) and cometary plus asteroidal (I) groups. Kresák proposes certain evolutionary tracks (due to Jupiter perturbation, collision, or radiation pressure) from asteroidal orbits to Earth-crossing orbits and investigates group I meteors for statistically significant subgroups compatible with this evolution. A number of unexpected and unexplained relationships emerge but none can be immediately applied as a criterion for asteroidal origin. Either it is not yet possible to formulate the problem sufficiently well or the group I orbits are heavily contaminated by material of cometary origin.

\section{REFERENCES}

Allen, H. J., and Baldwin, B. S., Jr. 1967, Frothing as an Explanation of the Acceleration Anomalies of Cometary Meteors. J. Geophys. Res. 72, 3483-3496.

Ayers, W. G., McCrosky, R. E., and Shao, C.-Y. 1970, Photographic Observations of 10 Artificial Meteors. Smithson. Astrophys. Observ. Spec. Rept. 317.

Baldwin, B. S., Jr., and Allen, H. J. 1968, A Method for Computing Luminous Efficiencies From Meteor Data. NASA TN D-4808.

Becker, D. G., and Friichtenicht, J. F. 1971, Measurement and Interpretation of the Luminous Efficiencies of Iron and Copper Simulated Micrometeors. Astrophys. J. 166, 699-716.

Ceplecha, Z. 1968, Discrete Levels of Meteor Beginning Height. Smithson. Astrophys. Observ. Spec. Rept. 279.

Cook, A. F. 1970, Discrete Levels of Beginning Height of Meteors in Streams. Smithson. Astrophys. Observ. Spec. Rept. 324.

Hawkins, G. S., and Southworth, R. B. 1961, Orbital Elements of Meteors. Smithson. Contrib. Astrophys. 4(3), 85-95.

Jacchia, L. G. 1955, The Physical Theory of Meteors. VIII Fragmentation as Cause of the Faint-Meteor Anomaly. Astrophys. J. 121, 521-527. 
Jacchia, L. G., Verniani, F., and Briggs, R. E. 1967, An Analysis of the Atmospheric Trajectories of 413 Precisely Reduced Photographic Meteors. Smithson. Contrib. Astrophys. 10(1), 1-139.

Jacchia, L. G., and Whipple, F. L. 1961, Precision Orbits of 413 Photographic Meteors. Smithson. Contrib. Astrophys. 4(4), 97-129.

Jones, S., and Kaiser, T. R. 1966, The Effects of Thermal Radiation, Conduction and Meteoroid Heat Capacity on Meteoric Ablation. Mon. Notic. Royal Astron. Soc. 133, 411-420.

Kresák, L. 1969, The Discrimination Between Cometary and Asteroidal Meteors. I. The Orbital Criteria. II. The Orbits and Physical Characteristics of Meteors. Bull. Astron. Inst. Czech. 20, 177-188, 231-251.

Kresák, L. 1970, On the Orbits of Bright Fireballs. Bull. Astron. Inst. Czech. 21, 1-9.

McCrosky, R. E. 1968, Orbits of Photographic Meteors. Physics and Dynamics of Meteors (eds., L. Kresák and P. M. Millman), pp. 265-279. D. Reidel. Dordrecht.

McCrosky, R. E., and Ceplecha, Z. 1970, Fireballs and the Physical Theory of Meteors. Bull. Astron. Inst. Czech. 21, 271-296.

McCrosky, R. E., and Posen, A. 1961, Orbital Elements of Photographic Meteors. Smithson. Contrib. Astrophys. 4(2), 15-84.

McCrosky, R. E., Posen, A., Schwartz, G., and Shao, C.-Y. 1971, Lost City Meteorite-Its Recovery and Comparison With Other Fireballs. J. Geophys. Res. 76(17), 4090-4108.

Whipple, F. L. 1954, Photographic Meteor Orbits and Their Distribution in Space. Astron. J. 59, $201-217$.

Whipple, F. L. 1967, On Maintaining the Meteoritic Complex. The Zodiacal Light and the Interplanetary Medium (ed., J. L. Weinberg), pp. 409-426. NASA SP-150. (Also Smithson. Astrophys. Observ. Spec. Rept. 239.)

\section{DISCUSSION}

KENKNIGHT: If you were to heat the outside layer of a meteorite, would it not tend to break up at lower stresses? Furthermore, are not the remnants we test in the lab anomalously strong because they have endured the atmospheric entry forces?

MCCROSKY: Thermal inertia and ablation govern the heating of a meteorite. The characteristic depth of heating-the depth at which the temperature decreases by $1 / e$-is a few millimeters. This temperature gradient does induce a tensile stress in the center of the body, but it cannot cause the breakup of large bodies.

As to your second question, I agree entirely that the atmosphere introduces a selection effect. However, the degree of atmospheric crushing required to produce the anomalous deceleration in meteors is, I think, unreasonable. In many cases the body must be broken into hundreds or thousands of fragments high in the atmosphere. 